\title{
REVIEW
}

\section{PRRT in high-grade gastroenteropancreatic neuroendocrine neoplasms (WHO G3)}

\author{
Halfdan Sorbye1,2, Grace Kong3,4 and Simona Grozinsky-Glasberg5 \\ 1Department of Oncology, Haukeland University Hospital, Bergen, Norway \\ 2Department of Clinical Sciences, University of Bergen, Bergen, Norway \\ ${ }^{3}$ Department of Molecular Imaging and Therapeutic Nuclear Medicine, Peter MacCallum Cancer Centre, Melbourne, Australia \\ 4 Sir Peter MacCallum Department of Oncology, The University of Melbourne, Melbourne, Australia \\ ${ }^{5}$ Neuroendocrine Tumor Unit, ENETS Center of Excellence, Department of Endocrinology and Metabolism, Hadassah-Hebrew University Medical Center, \\ Jerusalem, Israel
}

Correspondence should be addressed to H Sorbye: halfdan.sorbye@helse-bergen.no

\begin{abstract}
Peptide receptor radionuclide therapy (PRRT) is an established treatment for grade 1 and 2 gastroenteropancreatic neuroendocrine tumors with an increased uptake on somatostatin receptor imaging (SRI). Patients with metastatic high-grade (WHO G3) gastroenteropancreatic neuroendocrine neoplasms (NET G3 and NEC) represent a heterogeneous subgroup with poor prognosis and standard platinum-etoposide chemotherapy have limited therapeutic benefit. However, there is promising emerging evidence supporting the effectiveness of PRRT in SRI-positive G3 disease. A review search for studies reporting on PRRT in gastroenteropancreatic neuroendocrine neoplasms G3 was performed: four studies with more than ten cases were found. PRRT was mainly given as second- or third-line treatment in patients with progressive disease. Most patients had a pancreatic primary, 50\% had well-differentiated tumors, and most had a $\mathrm{Ki}-67<55 \%$. Three studies showed similar results with promising response rates (31-41\%) and disease control rates (69-78\%). Progression-free survival (11-16 months) and survival (22-46 months) were best concerning patients with a Ki-67<55\%. Progression-free survival was 19 months in NET G3, 11 months for lowNEC (Ki-67 $\leq 55 \%$ ) and 4 months for highNEC (Ki-67 >55\%). PRRT should be considered for patients with increased uptake on SRI, both in gastroenteropancreatic NET G3 cases and as well as in NEC cases with a Ki-67 $21-55 \%$. PRRT for NEC with a Ki-67 $>55 \%$ is less defined, but could be considered in highly selected cases after response to initial chemotherapy where all residual disease have high uptake on SRI. Dual tracer using 18F-FDG PET/CT and SRI provides important information for patient selection for PRRT in this heterogeneous complex high-grade disease.
\end{abstract}

\author{
Key Words \\ - peptide receptor \\ radionuclide therapy \\ - gastroenteropancreatic \\ - neuroendocrine carcinoma \\ - neuroendocrine tumors.
}

\section{Background}

\section{Peptide receptor radionuclide therapy (PRRT)}

Peptide receptor radionuclide therapy (PRRT) delivers highly localized radiation by targeting specific peptide receptors on tumor cells (Hicks et al. 2017).
This therapy has been established primarily for the treatment of un-resectable/metastatic well-differentiated neuroendocrine tumors (NET), which typically have high uptake on somatostatin receptor imaging (SRI), allowing targeting with PRRT. PRRT is comprised of three main 
Table 1 Nomenclature for clinical use on gastroenteropancreatic high-grade neuroendocrine neoplasms (NEN G3). ${ }^{a}$

\begin{tabular}{lll}
\hline NET G3 & Well-differentiated morphology, Ki-67 $>20 \%$ & lowNEC Ki-67 21-55\% \\
NEC & Poorly differentiated morphology, Ki-67 >20\% & highNEC Ki-67 >55\% \\
NEN G3 & Addressing both NET G3 and NEC or if differentiation is uncertain & \\
\cline { 3 - 4 }
\end{tabular}

aThe 2019 WHO classification separates NEC into small cell and large cell which at the moment has no clinical consequences and it does not separate NEC according to Ki-67 which does have clinical consequences.

components: a high activity radionuclide (177Lutetium or ${ }^{90}$ Yttrium), linked via a chelator (DTPA or DOTA) to a somatostatin receptor (SSR)-binding ligand which is typically a somatostatin analog (octreotide or octreotate). ${ }^{177} \mathrm{Lu}$-labeled PRRT is currently the radionuclide of choice for clinical use in most centers due to its lower toxicity compared to ${ }^{90}$ Y-labeled PRRT (Hicks et al. 2017). PRRT is administered intravenously, enabling the delivery of high doses of radiation selectively to sites of SSRexpressing tumors wherever they have spread throughout the body. Treatment scheduling typically consists of four cycles of ${ }^{177} \mathrm{Lu}-\mathrm{PRRT}$, each cycle given approximately 8 weeks apart (Kwekkeboom et al. 2005). SSR imaging (SRI) using ${ }^{111}$ In-Octreotide scintigraphy, or the more sensitive ${ }^{68} \mathrm{Ga}$-based PET/CT detects the presence of SSR on tumor cells and based on the degree of tumor uptake, is used to select patients for PRRT (Sundin et al. 2017). Although few formal, multicenter trials have been performed, there is relatively high consistency in the effectiveness of PRRT for gastroenteropancreatic (GEP) NET, with an encouraging $40 \%$ response rate $(\mathrm{RR}), 80 \%$ disease control rate (DCR) and long overall survival (OS) (Brabander et al. 2017, Hicks et al. 2017). The randomized NETTER-1 trial showed an increase in progression-free survival (PFS), RR, quality of life, and probably OS (follow-up ongoing) (Strosberg et al. 2017). PRRT is generally well tolerated with limited acute and medium-term toxicity. ${ }^{177} \mathrm{Lu}$-DOTA-octreotate (LuTathera) has recently obtained regulatory approval for patients with progressive metastatic grade 1-2 GEP NET.

\section{High-grade gastroenteropancreatic neuroendocrine neoplasms: NET G3 and NEC}

Gastroenteropancreatic (GEP) neuroendocrine neoplasms (NENs) are uncommon neoplasms accounting for $1.0-1.5 \%$ of all digestive cancers. In the new 2019 WHO classification of GEP NEN, the neuroendocrine tumor (NET) category is now used for well-differentiated tumors regardless of their proliferation index (G1-G3), whereas neuroendocrine carcinoma (NEC) category is used for poorly differentiated neoplasms. The high-grade GEP NEN G3 group (Ki-67 >20\%) is therefore now separated into two groups: NET G3 and NEC (Table 1) and is based on differences in morphology, biology, and genetic background (Heetfeld et al. 2015, Tang et al. 2016a, Milione et al. 2017, Sorbye et al. 2018). However, differentiation between the two and the pathological criteria for this subdivision in G3 NEN are not entirely straightforward and are evolving to more precise criteria. Clinically, NET G3 and NEC differ substantially from NET G1-G2. The prognosis is worse: metastatic disease is usually present at diagnosis, and the treatment of metastatic disease is different (Dasari et al. 2018, Sorbye et al. 2018). NEC do not usually respond to the standard treatments traditionally used in NET G1-G2 such as somatostatin analogs, everolimus and sunitinib (Garcia-Carbonero et al. 2016, Sorbye et al. 2019). Until recently, PRRT was generally not used for high-grade NENs. The assumption was that these tumors lacked SSR expression and the growth rate was too rapid to expect any benefit from PRRT. However, several recent retrospective studies have shown that high-grade tumors in a substantial number of cases have an increased uptake on SRI indicating high tumor SSR expression and that these patients seem to benefit from PRRT. A review of studies on the possible role of PRRT for high-grade GEP NENs was therefore performed.

\section{High-grade gastroenteropancreatic neuroendocrine tumors: NET G3}

Epidemiological data on NET G3 are very scarce and cases reported in studies are few and often with a stage mixture. The primary NET G3 tumor is, in contrast to NEC, mainly located in the pancreas and survival is significantly better (Velayoudom-Cephise et al. 2013, Basturk et al. 2015, Heetfeld et al. 2015, Milione et al. 2017, Sorbye et al. 2018). Median survival for metastatic patients is 33-41 months for GEP NET G3 compared to 11 months for GEP NEC treated with chemotherapy (Crippa et al. 2016, Tang et al. 2016a,b, Hijioka et al. 2017, Elvebakken et al. 2019). The optimal first-line palliative treatment for patients with metastatic NET G3 is unclear (Coriat et al. 2016). Response rate after platinum-based therapy seems much less (0-24\%) compared to NEC (40-50\%) 
(Heetfeld et al. 2015, Raj et al. 2017). PFS after platinumbased chemotherapy for NET G3 is short (2.4-5 months) indicating little benefit of platinum-based treatment, but also illustrating that NET G3 is a more aggressive disease than NET G2. A NET G2-like medical treatment strategy as tested in recent small retrospective studies may be more appropriate for patients with NET G3. Temozolomide/ capecitabine in patients with NET G3 tumors resulted in a disease control rate of 70\%, PFS 15 months and OS 22 months (Rogowski et al. 2019). Everolimus in patients with pancreatic NET G3 with a Ki-67 $\leq 55 \%$ resulted in a PFS of 6 months and OS of 28 months (Panzuto et al. 2017). Sunitinib gave a RR of $60 \%$ in ten pancreatic NET G3 patients, and treatment results did not differ from NET G1/2 patients (Mizuno et al. 2018).

\section{Gastroenteropancreatic neuroendocrine carcinoma: NEC}

NEC may arise throughout the gastroenteropancreatic system, but mainly in colon, rectum, stomach and pancreas (Dasari et al. 2018). GEP NEC are usually highly aggressive with a propensity for early metastatic disease (Sorbye et al. 2014, Walter et al. 2017). Patients with a Ki-67 21-55\% have a lower RR to platinum-based chemotherapy, but longer survival compared to cases with Ki-67 >55\% (Sorbye et al. 2013). A suggestion has been to subdivide the NEC category into a low NEC and a high NEC group based on a Ki-67 cut-off of 55\% (Table 1). In a high-grade GEP-NEN population, median survival was best for NET G3 (43.6 months), intermediate for NEC with Ki-67 21-55\% (24.5 months) and only 5.3 months for NEC cases with Ki-67 $\geq 55 \%$ (Milione et al. 2017). Although many metastatic NEC patients are initially responsive to systemic chemotherapy, the disease will progress essentially in all and the vast majority will eventually succumb to their disease. Most guidelines recommend the use of platinum-based chemotherapy combined with etoposide as first-line palliative treatment (Strosberg et al. 2010, Janson et al. 2014, Garcia-Carbonero et al. 2016). Results after first-line chemotherapy show a RR of 30-50\%, PFS of 4-6 months and OS of 11-16 months (Sorbye et al. 2013, Yamaguchi et al. 2014, Heetfeld et al. 2015, Walter et al. 2017). After first-line treatment, no further standard therapy has been established for GEP NEC. Temozolomide-based chemotherapy has resulted in a 33\% RR and a PFS of 6 months (Welin et al. 2011) and secondline FOLFIRI or FOLFOX result in short PFS ( $<3$ months) and OS ( $<6$ months) (Walter et al. 2017). Immunotherapy with checkpoint inhibitors as monotherapy has not been promising in GEP NEN G3 (Yao et al. 2018), whereas duo immunotherapy (ipilimumab+nivolumab) had tumor responses in two out of eight GEP NEN G3 cases (Patel et al. 2019).

\section{Somatostatin receptor imaging in high-grade GEP NEN (WHO G3)}

PRRT has previously not been recommended for GEP NEN G3 based on the rapid growth behavior and the expectance of loss of differentiation and low expression of somatostatin receptors. Previous research has demonstrated an inverse relationship between proliferation rate and SRI positivity (Ezziddin et al. 2006, 2014, Kayani et al. 2008). FDG uptake, a marker of glucose metabolism and proliferative activity, is usually high in GEP NEN G3 where small studies have shown a 75\% uptake in NET G3 and around 90\% in NEC cases (Walter et al. 2017, Sorbye et al. 2018). However, PRRT could be a relevant therapeutic option for NEN G3 as SRI positivity has been reported for both NET G3 and NEC and expression of somatostatin receptor 2A has been shown with immunohistochemistry (Table 2) (Konukiewitz et al. 2017). Recent observations suggest that uptake on SRI may be more frequent in G3 than initially anticipated (Table 2). Although data are limited and patient numbers small, SRI seems to be positive in $90 \%$ of NET G3 cases. SRI uptake is seen in approximately $40 \%$ in NEC; however, frequency varies greatly between studies and this number seems somewhat high. A bias of these observations is that SRI is probably more frequently performed in cases with a lower Ki-67 as was observed in the Nordic NEC study. A recent study of 11 GEP NEN G3 patients found that five patients with a positive SRI had no Rb1 loss, whereas four out of six with a negative SRI had loss of Rb1 expression (Liverani et al. 2018). Further prospective studies are needed to more accurately estimate the frequency of SSR expression detected by SRI for the general G3 NEN population.

\section{Methods}

A search for the use of PRRT in GEP NEN G3 was done in PubMed using the search terms: radionuclide therapy, PRRT, neuroendocrine tumors, neuroendocrine neoplasm, gastrointestinal, gastroenteropancreatic, neuroendocrine carcinoma and NET G3. Searches were limited to Englishlanguage articles. Studies reporting data only on G1 and G2 tumors or reporting on less than ten GEP NEN G3 cases 
Table 2 Studies reporting results on somatostatin receptor imaging (uptake > liver) in patients with gastroenteropancreatic high-grade neuroendocrine neoplasms (NEN G3).

\begin{tabular}{|c|c|c|c|c|c|}
\hline & $n$ & $\begin{array}{c}n \text { with } \\
\text { SRI }\end{array}$ & $\begin{array}{c}\text { NEN G3 } \\
\text { SRI uptake > liver NEN G3 } \\
\end{array}$ & $\begin{array}{c}\text { NEN G3 } \\
\text { SRI uptake }>\text { liver NET G3 }\end{array}$ & $\begin{array}{c}\text { NEN } \\
\text { SRI uptake }>\text { liver NEC }\end{array}$ \\
\hline Welin et al. 2011 & 25 & $20^{a}$ & $8 / 20(40 \%)$ & & \\
\hline Sorbye et al. 2013 & 305 & $182^{\mathrm{a}}$ & $68 / 182(37 \%)$ & & \\
\hline Velayoudom-Cephise et al. 2013 & 28 & $14^{a}$ & 10/14 (71\%) & $7 / 8(88 \%)$ & $3 / 6(50 \%)$ \\
\hline Heetfeld et al. 2015 & 204 & $82^{c}$ & $44 / 82(54 \%)$ & $21 / 24(92 \%)$ & 23/58 (40\%) \\
\hline Bongiovanni et al. 2015 & 20 & $19 \mathrm{~b}$ & $7 / 19(37 \%)$ & & \\
\hline Walter et al. 2017 & 253 & $40^{a}$ & & & $15 / 40(38 \%)$ \\
\hline Raj et al. 2017 & 45 & $27^{a}$ & 19/27 (70\%) & $13 / 15(87 \%)$ & $6 / 12(50 \%)$ \\
\hline
\end{tabular}

aSomatostatin receptor scintigraphy, bGallium-68 PET/CT, cMethod not specified.

$\mathrm{SRI}$, somatostatin receptor imaging.

were excluded. Four studies with $>10$ cases were found reporting results from PRRT in GEP NEN G3 patients.

\section{PRRT in GEP NEN G3}

We identified four studies with $>10$ cases reporting on PRRT outcomes in GEP NEN G3 patients (Thang et al. 2017, Nicolini et al. 2018, Carlsen et al. 2019, Zhang et al. 2019).

The first study by Nicolini et al. differs in several aspects from the other three studies described (Nicolini et al. 2018). In this study, $13 / 33$ patients had a tumor Ki-67 between 2 and 20\%, whereas in only 20/33 patients the Ki-67 was more than $20 \%$. This probably explains why their reported survival for the whole group in this study seems to be longer than reported in the other three studies where only patients with a Ki-67 >20\% were included (Fig. 2 and Table 3). The Nicolini study does not specify if the patients had progressive disease prior to PRRT. Differentiation is described, however not according to the 2017 WHO classification, as seven NEC cases are reported to be well-differentiated. Data on hormonal hypersecretion were not provided, but as many as $42 \%$ had clinical symptoms including flushing and diarrhea, which is unusual in G3 patients. The study reported a low RR of 6\%, PFS 23 months and OS 60 months. Patients with a Ki-67 below 35\% had a much better PFS (26.3 vs 6.8 months) and OS (53 months vs 12.6 months). As the population included in this study seems to be very different from the patients included into the other studies, comparisons are difficult.

In the study of Thang et al., 28 patients were included, $89 \%$ with progressive disease (Thang et al. 2017). Two patients had a bronchial primary tumor, the rest having GEP NEN of mainly pancreatic origin (61\%) or unknown primary. Ki- 67 was $\leq 55 \%$ in $79 \%$ of the cases. The study did not specify if the morphology was well- or poorly differentiated. Treatment was mainly given as second (45\%) or third line (29\%). The response rate (per CT/MRI) at 3 months post PRRT was 35\%. For patients with a Ki-67 $\leq 55 \%$, PFS was 12 months and OS 46 months. For patients with a Ki-67 $>55 \%$, PFS was 4 months and OS 7 months. The disease control rate (DCR) was $74 \%$.

In the study of Zhang et al., 69 patients were included (Zhang et al. 2019). All patients had GEP or unknown primary, most had a pancreatic primary (67\%). All patients had progressive disease. Fifty-three cases (77\%) had Ki-67 $\leq 55 \%$. Morphology results were available for $42 / 69$ cases; 28 (67\%) had poorly differentiated morphology.

Table 3 Patients characteristics, treatment response and survival after PRRT in patients with high-grade gastroenteropancreatic neuroendocrine neoplasms in three referral studies. ${ }^{a}$

\begin{tabular}{|c|c|c|c|c|c|c|c|c|}
\hline & \multirow[b]{2}{*}{$n$} & \multirow[b]{2}{*}{ Pancreatic primary } & \multirow[b]{2}{*}{$\mathbf{R R}$} & \multirow[b]{2}{*}{ DCR } & \multicolumn{2}{|c|}{ PFS } & \multicolumn{2}{|c|}{ os } \\
\hline & & & & & Low Ki-67 & High Ki-67 & Low Ki-67 & High Ki-67 \\
\hline Thang et al. 2017 & 28 & $61 \%$ & $35 \%$ & $74 \%$ & 12 months & 4 months & 46 months & 7 months \\
\hline Zhang et al. 2019 & 69 & $67 \%$ & $31 \%$ & $78 \%$ & 11 months & 4 months & 22 months & 7 months \\
\hline Carlsen et al. 2019 & 149 & $60 \%$ & $42 \%$ & $69 \%$ & 16 months & 6 months & 31 months & 9 months \\
\hline
\end{tabular}

All studies report retrospective data and some of the data are based on a very low number of patients.

aMainly given as second- and third-line therapy.

DCR, disease control rate; High Ki-67, Ki-67 >55\%; Low Ki-67, Ki-67 21-55\%; OS, overall survival; PFS, progression free survival; RR, response rate on CT.

https://erc bioscientifica com

https://doi.org/10.1530/ERC-19-0400
(C) 2020 Society for Endocrinology Published by Bioscientifica Ltd. Printed in Great Britain 


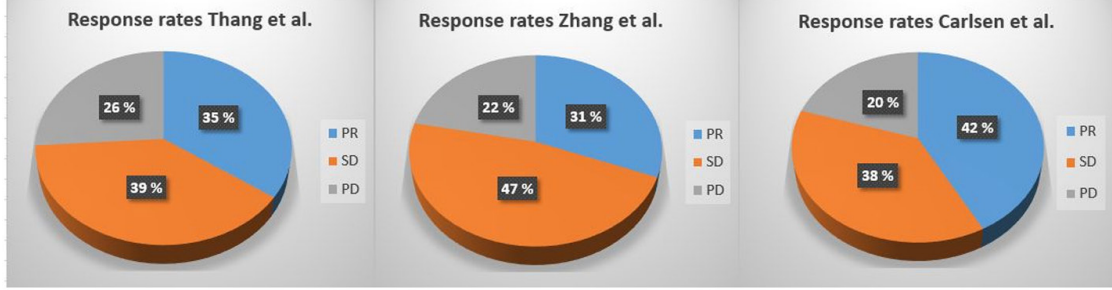

\section{Figure 1}

Response rates after PRRT in patients with

high-grade gastroenteropancreatic neuroendocrine neoplasms according to three studies. Evaluable patients: $n=23$ (Thang), $n=55$ (Zhang), $n=114$ (Carlsen).

Hormonal levels or functional secretory symptoms were not specified. Treatment was mainly given as second (36\%) or third line $(41 \%)$. Response rate (RECIST) for evaluable patients 3 months post-PRRT was 31\% and additional $47 \%$ had stable disease (SD). DCR was 78\%. For patients with a Ki-67 $\leq 55 \%$, PFS was 11 months and OS 22 months. For patients with a Ki-67 $>55 \%$, PFS was 4 months and OS 7 months. A mismatch pattern with high uptake on SRI and no or minor uptake on FDG-PET was associated with a better long-term prognosis.

Finally, in the study of Carlsen et al., 149 patients were included (Carlsen et al. 2019). All patients had GEP or unknown primary with a dominance of abdominal metastases; most had a pancreatic primary (60\%). 104 patients had documented radiological progressive disease at start of PRRT. 125 cases (84\%) had Ki-67 $\leq 55 \%$. Morphology results were available for $129 / 149$ cases; $62(42 \%)$ had poorly differentiated morphology and 60 (40\%) had well-differentiated morphology. Treatment was mainly given as second (42\%) or third/later lines (38\%). Response rate (RECIST) was 42\%, an additional 38\% had SD and DCR was 69\%. RR did not differ according to differentiation or Ki-67. For patients with a Ki-67 $\leq 55 \%$, PFS was 16 months and OS 31 months. For patients with a Ki-67 >55\%, PFS was 6 months and OS 9 months. Survival was better for patients with well-differentiated tumors compared to poorly differentiated neoplasms (PFS 19 vs 8 months and OS 44 months vs 19 months). In a multivariate analyses performance status (PS), elevated plasma LDH and ALP levels were significant predictors for PFS and OS, whereas age was significant for PFS and differentiation for OS.

The populations included in these last three studies (Thang et al. 2017, Carlsen et al. 2019, Zhang et al. 2019) seem quite similar: $2 / 3$ of cases had pancreatic primaries, Ki-67 was mainly below 55\% (78-84\%), median age 57-63 years and PRRT given mainly as second or third line treatment. There were some differences between the studies. The study of Thang et al. did not report on morphology data (NET G3 vs NEC). The study of Zhang et al. had mainly patients with poor differentiation on morphology (41\%), but as many as $39 \%$ did not have morphology data available. In Carlsen et al., 40\% had well-differentiated tumors, $42 \%$ had poorly differentiated morphology and only 12\% lacked morphology data. Another variable was the use of radiosensitising chemotherapy for 20/28 patients (71\%) in Thang et al. compared to 22/69 (32\%) in Zhang et al., whereas only six patients (4\%) received radiosensitisers in Carlsen et al. However, in general, the population and treatment seem quite similar and cross trial comparisons with caution seem reasonable. The results of these three studies are astonishingly similar concerning RR, PFS and OS. RRs were as high as $34-47 \%$ and only $20-26 \%$ had immediate disease progression on PRRT (Fig. 1 and Table 3). All studies showed a better PFS and OS for patients with a lower Ki-67. For patients with a Ki-67 21-55\%, PFS was 11-16 months and OS 22-46 months (Table 3). For patients with a Ki-67 >55\%, PFS was 4-6 months and OS 7-9 months.

Noteworthy, the Carlsen et al. study also presented NEN G3 data separated into three categories: NET G3, lowNEC (Ki-67 21-55\%) and highNEC (Ki-67 >55\%). The results show a clear separation according to these three groups and support recommendations that data from high-grade NENs should be analysed also by NEC subgroups. Response rate for NET G3 and lowNEC were similar (42-43\%), but immediate disease progression was more frequent in lowNEC than NET G3 (26 vs 7\%) (Fig. 2A). Although based only on few patient data, the highNEC group displayed a completely different treatment response pattern with a $50 \%$ chance of either a response or immediate progression. PFS was 19 months in NET G3, 11 months for lowNEC and 4 months for highNEC (Fig. 2B), whereas survival was 44 months for NET G3, 22 months for lowNEC and 9 months for highNEC (Fig. 2C).

\section{Toxicity}

For all selected studies, toxicity of PRRT in GEP NEN G3 did not seem different from large retrospective studies on grade 1-2 NET patients given PRRT (Imhof et al. 2011, Kwekkeboom et al. 2008). The three studies did not record any myelodysplasia or leukemia cases. Thang et al. reported grade 3-4 toxicity with lymphopenia (five cases) 

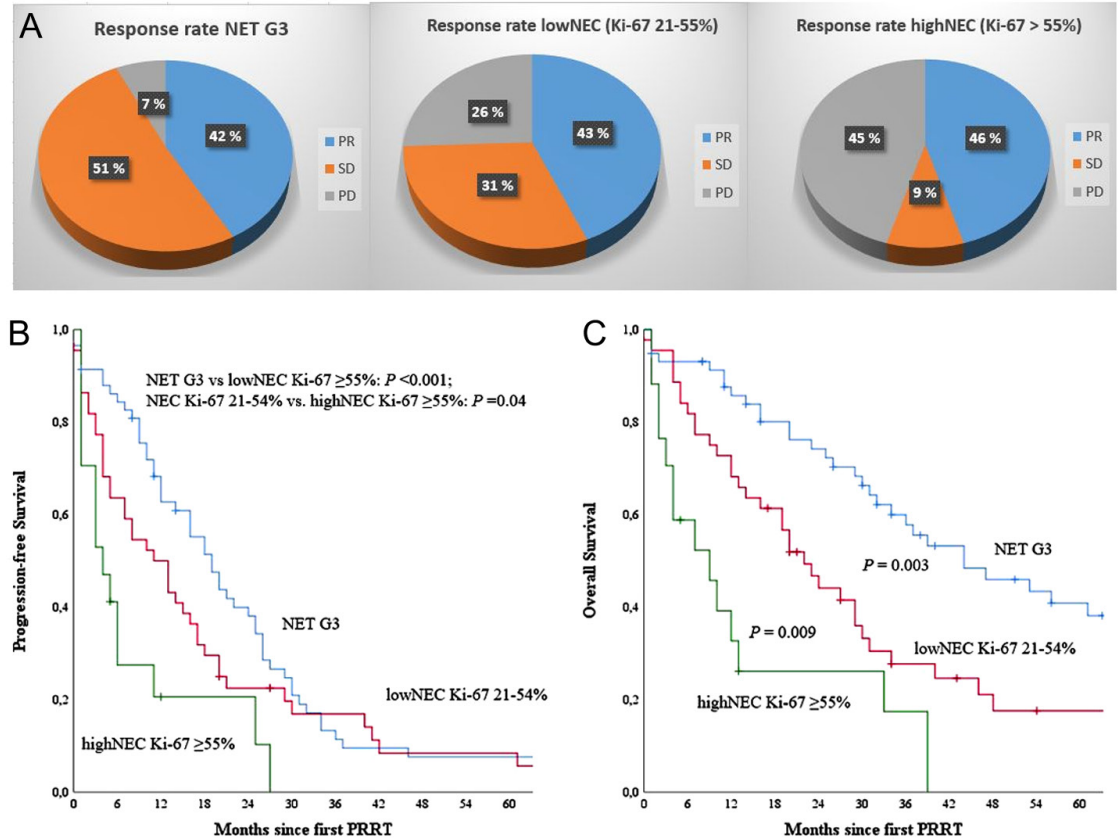

\begin{abstract}
Figure 2
Response rate (A), progression-free survival (B) and overall survival (C) after PRRT in 149 patients with high-grade gastroenteropancreatic neuroendocrine neoplasms according to three subgroups: NET G3, lowNEC (Ki-67 21-55\%) and highNEC (Ki-67 >55\%). $n=58$ for NET G3, $n=44$ for lowNEC and $n=17$ for highNEC. (B and C) data from Carlsen et al. (2019).
\end{abstract}

and thrombocytopenia (five cases). No grade 3-4 renal impairment or hematological toxicity was reported by Zhang et al. Carlsen et al. reported grade 3-4 acute toxicity in 12 cases (9 hematological and 3 renal) and grade 3-4 long-term toxicity in 15 cases (11 hematological and 3 renal). In studies on PRRT in GEP NET grade 1-2, subacute grade 3 or 4 hematological toxicities have been reported to occur in up to $11 \%$. Severe renal complications are reportedly uncommon (1.5\%) (Hicks et al. 2017).

\section{Are these three study cohorts comparable to the general high-grade NEN G3 population?}

Randomized studies to assess the benefit of specific treatments are often not feasible for very rare diseases such as GEP NEN G3. Large retrospective datasets may initially be the only reasonable source to build treatment decisions. However, retrospective datasets have several limitations including mainly the variability of patient selection, inclusion criteria, treatment and follow-up protocols. The main possible difference between the three study cohorts and the general GEP NEC population is probably the presence of a high uptake on SRI. However, as shown in Table 2, there may be more NEC patients with a high uptake on SRI than previously expected. The SRI data summarized in Table 2, as well as emerging from the three referral studies would strongly support consideration of SRI in patients with G3 NEN, particularly for NET G3 or lowNEC given the potential effective theranostic approach in these patients and warrants further prospective evaluation. The three referral studies consist only of few patients with a high Ki-67 (>55\%), many patients with well-differentiated tumor morphology (NET G3) (usually $5-15 \%$ in other studies) and many pancreatic NEC cases (67 vs 25\% in other NEC populations) (Heetfeld et al. 2015, Leoncini et al. 2017, Walter et al. 2017, Sorbye et al. 2018). The presence of pancreatic primaries in $66 \%$ of NET G3 cases fits, however, well with prior studies. In the Carlsen et al. study, the prognostic factors for benefit of PRRT included poor PS and elevated LDH, similar to prior studies (Sorbye et al. 2013, Lamarca et al. 2017). Elevated hormonal levels or functional secretory symptoms were seen in as many as one-third of patients in the Thang study, whereas the two other studies did not register hormonal symptoms. Hormonal symptoms are found only in 1-3\% for NEC and 14\% for NET G3 (Heetfeld et al. 2015, Walter et al. 2017, Sorbye et al. 2018). Despite some potential differences between the patients in these three studies compared to the general NEN G3 population, patient selection based on high uptake on SRI remains relevant for PRRT as a promising effective therapeutic approach for NEN G3 patients.

\section{What is the true benefit of PRRT in NEN G3}

As shown previously, the patients from the three studies were selected based on high SSR expression as indicated by positive uptake on SRI. High SRI uptake not only (c) 2020 Society for Endocrinology Published by Bioscientifica Ltd. Printed in Great Britain 
enables the potential for a theranostics approach, but it also likely indicates a good prognostic factor for GEP NEN G3 patient, probably reflecting a more well-differentiated neoplasm with less aggressive tumor growth. Comparison of results with other GEP NEN G3 studies of different therapeutic modalities must therefore be done with caution. Results from the three studies are mainly based on patients with pancreatic primary and most patients had NET G3 or lowNEC (Ki-67 <55\%). However, the PFS and OS seem impressive as second- and third-line therapy, especially for the NET G3 and lowNEC group. Importantly, the favorable responses (RR, DCR and PFS) from PRRT, despite the high number of patients with documented progressive disease, indicate that PRRT is an active treatment for these patients. Platinum-based firstline chemotherapy for metastatic NET G3 have given RR of $0-24 \%$, PFS 3-5 months and OS 30 months. PRRT given as mainly second or third line in NET G3 gave in comparison a RR 42\%, PFS 19 months and OS 44 months - clearly indicting a benefit of PRRT in NET G3 (Table 4) (Carlsen et al.). For NEN G3 with a Ki-67 $<55 \%$ and specifically the lowNEC subgroup available data suggest a possible substantial benefit of PRRT for this subgroup (Table 4). The outcomes of PRRT in GEP NEN G3 with a $\mathrm{Ki}-67>55 \%$ are based on limited number of cases and a possible benefit is therefore difficult to assess.

\section{Which GEP NEN G3 patients should be offered PRRT?}

Pending further research, the crucial clinical questions are to decide which GEP NEN G3 patients should be offered PRRT, when in the treatment sequence it should be used and, importantly, to identify the patients who are unlikely to benefit from PRRT. ENETS guidelines recommend that PRRT can be considered in SRI-positive NET G3 (Garcia-Carbonero et al. 2016), whereas the National Comprehensive Cancer Network (NCCN) recommend only the use of PRRT in GEP NEN when Ki-67 is $\leq 20 \%$ (NCCN 2019). PRRT can be effective for patients with FDG-avid disease provided high SRI uptake is also retained at all disease sites (Kashyap et al. 2015). However, if there are spatially discordant (FDG positive/ SRI negative) lesions, PRRT alone is unable to target the discordant poorly differentiated components, and other systemic options should be considered in these patients (Chan et al. 2017, Thang et al. 2017). Therefore, dual tracer whole body PET/CT imaging provides important prognostic information, disease characterization and should be considered as a pre-requisite for patients with G3 NEN to guide management and selection for PRRT, especially given the potential heterogeneity and otherwise very poor prognosis in patients with high-grade poorly differentiated tumors.

In our opinion, PRRT should be considered for all GEP NEN G3 cases with well-differentiated tumors (NET G3) and for all GEP NEN G3 cases with a Ki- $67<55 \%$ regardless of the differentiation as long as all lesions show high uptake on SRI. Figure 3 shows a pancreatic NET G3 case with an impressive response sustained at 26 months from start of PRRT. As NENs are typically treated in a sequential fashion with a variety of therapeutic lines, it is important to understand when it could be utilized (Kong \& Hicks 2019). The optimal sequencing for these patients is yet unknown, but given the poor response with chemotherapy in NET G3 and lowNEC (and all NEN G3 with a Ki-67 $<55 \%$ ) and the encouraging responses with PRRT in these groups, it may support the use of PRRT in a more upfront setting. Concerning the use of PRRT in NEN G3 cases with

Table 4 Results of chemotherapy compared to PRRT in in patients with metastatic high-grade gastroenteropancreatic neuroendocrine neoplasms (NEN G3).

\begin{tabular}{|c|c|c|c|c|c|c|c|c|c|}
\hline & \multicolumn{3}{|c|}{ 1-line chemotherapy } & \multicolumn{3}{|c|}{ PRRT (mainly 2/3-line) } & \multicolumn{3}{|c|}{ 2-line chemotherapyc } \\
\hline & RR & PFS & OS & RR & PFS & OS & RR & PFS & OS \\
\hline NET G3a & $0-24 \%$ & 3-5 months & 33 months & $42 \%$ & 19 months & 44 months & & & \\
\hline lowNECa & $25 \%$ & 5 months & 11 months & $43 \%$ & 11 months & 22 months & $16-24 \%$ & $<3$ months & $<6$ months \\
\hline highNECa & $44 \%$ & 5 months & 11 months & $46 \%$ & 4 months & 9 months & $16-24 \%$ & $<3$ months & $<6$ months \\
\hline $\begin{array}{l}\text { NEN G3b } \\
\text { Ki-67 21-55\% }\end{array}$ & $15 \%$ & 4 months & 14 months & $34-35 \%$ & $\begin{array}{l}11-12 \\
\text { months }\end{array}$ & $22-46$ months & $16-24 \%$ & $<3$ months & $<6$ months \\
\hline $\begin{array}{l}\text { NEN G3b } \\
\text { Ki-67 >55\% }\end{array}$ & $42 \%$ & 4 months & 10 months & $0-33 \%$ & 4 months & 7 months & $16-24 \%$ & $<3$ months & $<6$ months \\
\hline
\end{tabular}

All study data are retrospective and some of the data are based on a very low number of patients. All cases given PRRT had a high uptake on SRI. aPRRT data from Carlsen et al. and 1-line chemotherapy data from Heetfeld et al., Raj et al. and Elvebakken et al. bPRRT data from Thang et al. and Zhang et al., 1-line chemotherapy data from Sorbye et al. 2013. cResults for NEC as a whole group from Walter et al. and Sorbye et al. 2013. highNEC, Ki-67 >55\%; lowNEC, Ki-67 21-55\%; OS, overall survival; PFS, progression-free survival; RR, response rate on CT.

(c) 2020 Society for Endocrinology Published by Bioscientifica Ltd. Printed in Great Britain 


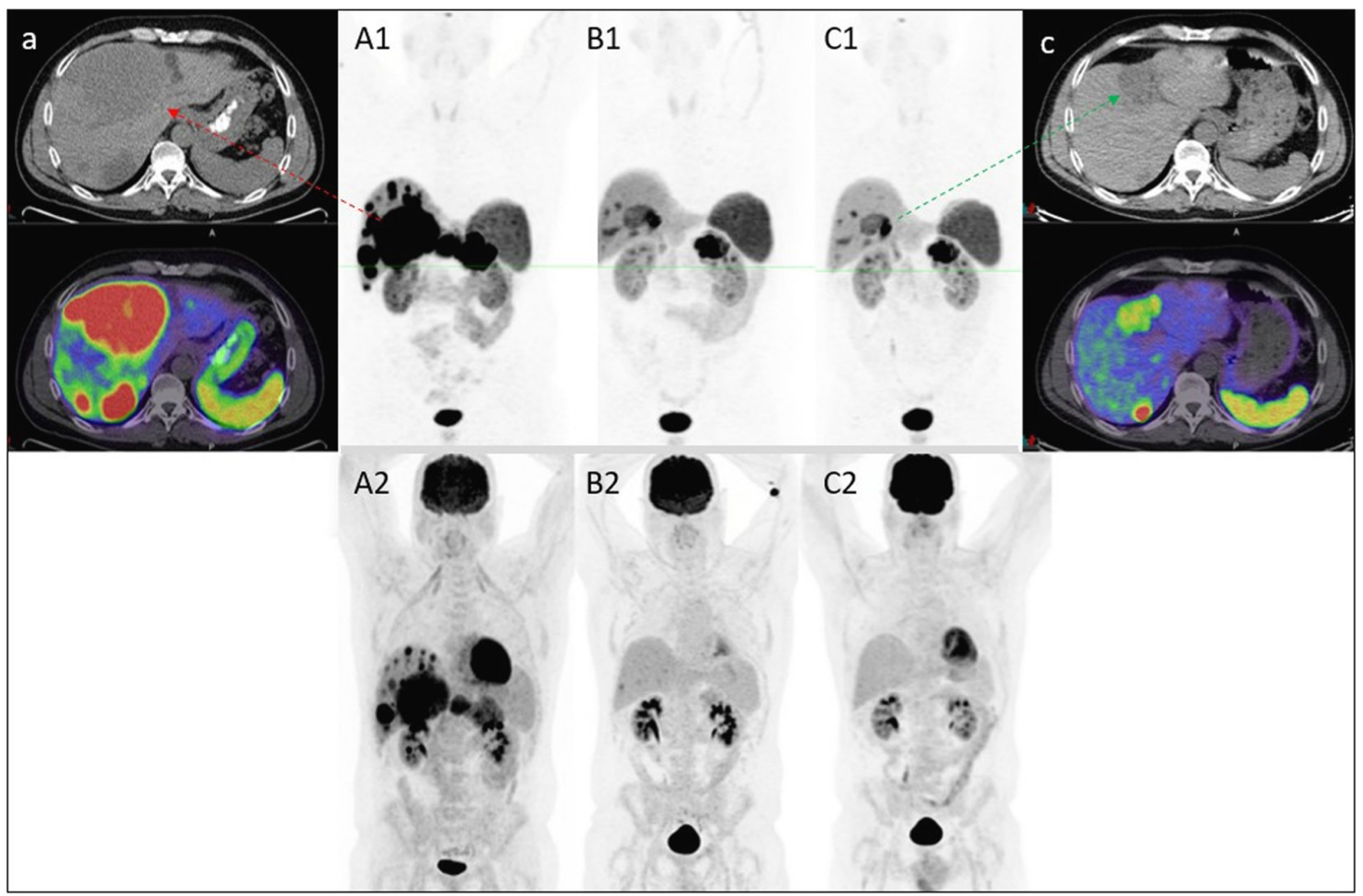

\section{Figure 3}

A 50-year-old male with pancreatic NET G3 (Ki-67 50\%) and multiple liver and nodal metastases. Baseline imaging showed high somatostatin receptor (SSR) expression at all disease sites (A1: 68Ga-DOTATATE PET/CT), with concordant high metabolic activity (A2: 18F-FDG PET/CT). Given high SSR expression and intolerance to carboplatin/etoposide, induction PRRT was given. 3 months post-treatment: significant reduction of tumor burden on 68Ga-DOTATATE PET/CT (B1) and near complete metabolic response (B2). Response sustained at 26 months from start of PRRT (C1 and C2). Significant tumor regression highlighted in an index liver lesion (a: pre-treatment CT component of 68Ga-DOTATATE PET/CT, and trans-axial fused images; C: post-therapy images).

a Ki-67 $>55 \%$, we advise extreme caution until more data are available. It seems justifiable in highly selected patients responding to systemic treatment and performing better than expected. However, in rapidly progressing patients and NEC patients with reduced performance status (PS $>1$ ), PRRT should be used with discretion. In NEC cases PRRT is usually administered at tumor progression and after classic chemotherapy; however, one could expect it would work better as a consolidation treatment immediately after induction chemotherapy. Re-treatment with PRRT, defined as a re-challenge after four initial PRRT cycles with initial response, is feasible and effective in G1/G2 NET cohorts (Rudisile et al. 2019). In a recent report on 168 GEP-NEN patients, 7/9 patients with WHO grade 3 tumor (Ki-67 20-30\%) responded to PRRT re-treatment with a PFS of 13.4 months (van der Zwan et al. 2019). The authors suggest that re-treatment with PRRT is an option in a subgroup of patients with GEP-NEC with progressive disease following initial PRRT, primarily in those with a Ki-67 below 30\%. In Thang et al., 11 of 28 NEN G3 patients received further PRRT due to recrudescent disease after initial response, with a median PFS of 12 months and a median OS of 46 months.

\section{Future aspects}

There is an urgent need for prospective trials to confirm efficacy of available treatments, to guide sequencing and to explore QOL parameters to balance toxicity with effectiveness in treatments for GEP NEN G3 patients. Prospective data to establish PRRT efficacy is warranted. Future developments should also focus on a more personalized approach based on better characterization of disease and individual patient factors which is relevant in the context of this heterogeneous and complex disease (Kong \& Hicks 2019). Multiple potential new developments are underway to further enhance efficacy of PRRT including changes of radioligand, use of dosimetry and combination therapy with different agents (Brabander et al. 2019). Theoretically, peptide receptor chemoradionuclide therapy (PRCRT), a combination of PRRT and a radiosensitizer, 
should be useful in G3 patients. Several studies have used concomitant chemotherapy including infusional 5FU, oral capecitabine or capecitabine+temozolomide with favorable responses and acceptable toxicity for patients with metastatic NEN (Kong et al. 2014, Kashyap et al. 2015, Claringbold \& Turner 2016). Further prospective studies comparing PRCRT against PRRT alone are underway. The optimal PRRT scheduling might be different for highgrade NENs. The rapid progression in these high-grade neoplasms could benefit from closer spacing of treatment cycles to avoid early repopulation of cancer cells between treatments. Further research is needed to study differences between NEC cases with and without SRI uptake, for example, vs other treatment responses or in relation to their genetic profile. More prospective data is also needed to establish the role of molecular imaging, which should be incorporated in prospective G3 trials. A prospective trial to assess PRRT vs CAPTEM in patients with G3 NEN $<55 \%$ would be clinically relevant. The NETTER- 2 study has essentially the same treatment arms as NETTER-1, but for higher grade 2 and grade 3 cases (Ki-67<55\%) with the comparative arms PRRT+ somatostatin analogs vs higher dose somatostatin analogs (https://clinicaltrials.gov/ct2/ show/NCT03972488).

\section{Conclusion}

PRRT for high-grade gastroenteropancreatic neuroendocrine neoplasms with a high uptake on SRI have shown promising response rates, disease control rates, progression-free survival and overall survival. Until further data are available, PRRT should be considered for all NET G3 cases and NEC cases with a Ki-67 21-55\% with high uptake on SRI. PRRT for NEC with a Ki-67 $>55 \%$ should only be considered in highly selected patents and used with caution. Dual tracer molecular imaging plays a major role in patient selection for PRRT in the context of G3 NEN. Further prospective studies are warranted to confirm the efficacy of PRRT and to guide sequencing of therapies, with the aims of improving patient outcome in this poor prognostic patient group.

\section{Declaration of interest}

H S has received research support from Novartis, Amgen, Ipsen and honoraria from Novartis, Ipsen, Pfizer, Keocyt, AstraZeneca, Roche, Amgen, Merck, Shire and Celgene. G K is supported by a Foundation Discovery Partner Fellows award from the Peter MacCallum Cancer Foundation. S G $G$ has received research support from Novartis and Ipsen and honoraria from Novartis, Ipsen, Pfizer, AAA and Lexicon.

(c) 2020 Society for Endocrinology Published by Bioscientifica Ltd. Printed in Great Britain

\section{Funding}

This work did not receive any specific grant from any funding agency in the public, commercial or not-for-profit sector.

\section{References}

Basturk O, Yang Z, Tang LH, Hruban RH, Adsay V, Mccall CM, Krasinskas AM, Jang KT, Frankel WL, Balci S, et al. 2015 The highgrade (WHO G3) pancreatic neuroendocrine tumor category is morphologically and biologically heterogenous and includes both well differentiated and poorly differentiated neoplasms. American Journal of Surgical Pathology 39 683-690. (https://doi.org/10.1097/ PAS.0000000000000408)

Brabander T, Van Der Zwan WA, Teunissen JJM, Kam BLR, Feelders RA, De Herder WW, Van Eijck CHJ, Franssen GJH, Krenning EP \& Kwekkeboom DJ 2017 Long-term efficacy, survival, and safety of [(177)Lu-DOTA(0), Tyr(3)]octreotate in patients with gastroenteropancreatic and bronchial neuroendocrine tumors. Clinical Cancer Research 23 4617-4624. (https://doi. org/10.1158/1078-0432.CCR-16-2743)

Brabander T, Nonnekens J \& Hofland J 2019 The next generation of peptide receptor radionuclide therapy. Endocrine-Related Cancer $\mathbf{2 6}$ C7-C11. (https://doi.org/10.1530/ERC-19-0186)

Bongiovanni A, Riva N, Ricci M, Liverani C, La Manna F, De Vita A, Foca F, Mercatali L, Severi S, Amadori D \& Ibrahim T 2015 First-line chemotherapy in patients with metastatic gastroenteropancreatic neuroendocrine carcinoma. OncoTargets and Therapy 8 3613-3619.

Carlsen EA, Fazio N, Granberg D, Grozinsky-Glasberg S, Ahmadzadehfar H, Grana CM, Zandee WT, Cwikla J, Walter MA, Oturai PS, et al. 2019 Peptide receptor radionuclide therapy in gastroenteropancreatic NEN G3: a multicenter cohort study. Endocrine-Related Cancer 26 227-239. (https://doi.org/10.1530/ERC18-0424)

Chan DL, Pavlakis N, Schembri GP, Bernard EJ, Hsiao E, Hayes A, Barnes T, Diakos C, Khasraw M, Samra J, et al. 2017 Dual somatostatin receptor/FDG PET/CT imaging in metastatic neuroendocrine tumours: proposal for a novel grading scheme with prognostic significance. Theranostics 7 1149-1158. (https://doi. org/10.7150/thno.18068)

Claringbold PG \& Turner JH 2016 Pancreatic neuroendocrine tumor control: durable objective response to combination Lu-177-octreotatecapecitabine-temozolomide radiopeptide chemotherapy. Neuroendocrinology 103 432-439. (https://doi.org/10.1159/000434723)

Coriat R, Walter T, Terris B, Couvelard A \& Ruszniewski P 2016 Gastroenteropancreatic well-differentiated grade 3 neuroendocrine tumors: review and position statement. Oncologist 21 1191-1199. (https://doi.org/10.1634/theoncologist.2015-0476)

Crippa S, Partelli S, Bassi C, Berardi R, Capelli P, Scarpa A, Zamboni G \& Falconi M 2016 Long-term outcomes and prognostic factors in neuroendocrine carcinomas of the pancreas: morphology matters. Surgery 159 862-871. (https://doi.org/10.1016/j.surg.2015.09.012)

Dasari A, Mehta K, Byers LA, Sorbye H \& Yao JC 2018 Comparative study of lung and extrapulmonary poorly differentiated neuroendocrine carcinomas: a SEER database analysis of 162,983 cases. Cancer 124 807-815. (https://doi.org/10.1002/cncr.31124)

Elvebakken H, Perren A, Scoazec JY, Tang L, Fiederspiel B, Vestermark L, Zlobec I, Hjortland G, Langer SW, Gronbeck H, et al. 2019 A consensus developed morphological re-evaluation of 196 cases with metastatic high-grade gastroenteropancreatic neuroendocrine neoplasms (GEP NEN G3). Neuroendocrinology 108 (Suppl 1) abstract H09. (https://doi.org/10.1159/000498996)

Ezziddin S, Logvinski T, Yong-Hing C, Ahmadzadehfar H, Fischer HP, Palmedo H, Bucerius J, Reinhardt MJ \& Biersack HJ 2006 Factors 
predicting tracer uptake in somatostatin receptor and MIBG scintigraphy of metastatic gastroenteropancreatic neuroendocrine tumors. Journal of Nuclear Medicine 47 223-233.

Ezziddin S, Khalaf F, Vanezi M, Haslerud T, Mayer K, Al Zreiqat A, Willinek W, Biersack HJ \& Sabet A 2014 Outcome of peptide receptor radionuclide therapy with $177 \mathrm{Lu}$-octreotate in advanced grade $1 / 2$ pancreatic neuroendocrine tumours. European Journal of Nuclear Medicine and Molecular Imaging 41 925-933. (https://doi.org/10.1007/ s00259-013-2677-3)

Garcia-Carbonero R, Sorbye H, Baudin E, Raymond E, Wiedenmann B, Niederle B, Sedlackova E, Toumpanakis C, Anlauf M, Cwikla JB, et al. 2016 Enets consensus guidelines for high-grade gastroenteropancreatic neuroendocrine tumors and neuroendocrine carcinomas. Neuroendocrinology 103 186-194. (https://doi. org/10.1159/000443172)

Heetfeld M, Chougnet CN, Olsen IH, Rinke A, Borbath I, Crespo G, Barriuso J, Pavel M, O'toole D, Walter T, et al. 2015 Characteristics and treatment of patients with G3 gastroenteropancreatic neuroendocrine neoplasms. Endocrine-Related Cancer 22 657-664. (https://doi.org/10.1530/ERC-15-0119)

Hicks RJ, Kwekkeboom DJ, Krenning E, Bodei L, Grozinsky-Glasberg S, Arnold R, Borbath I, Cwikla J, Toumpanakis C, Kaltsas G, et al. 2017 Enets consensus guidelines for the standards of care in neuroendocrine neoplasia: peptide receptor radionuclide therapy with radiolabeled somatostatin analogues. Neuroendocrinology 105 295-309. (https://doi.org/10.1159/000475526)

Hijioka S, Hosoda W, Matsuo K, Ueno M, Furukawa M, Yoshitomi H, Kobayashi N, Ikeda M, Ito T, Nakamori S, et al. 2017 Rb loss and KRAS mutation are predictors of the response to platinum-based chemotherapy in pancreatic neuroendocrine neoplasm with grade 3 : a japanese multicenter pancreatic Nen-G3 study. Clinical Cancer Research 23 4625-4632. (https://doi.org/10.1158/1078-0432.CCR-163135)

Imhof A, Brunner P, Marincek N, Briel M, Schindler C, Rasch $\mathrm{H}$, Macke HR, Rochlitz C, Muller-Brand J \& Walter M A 2011 Response, survival, and long-term toxicity after therapy with the radiolabeled somatostatin analogue [Y-90-DOTA]-TOC in metastasized neuroendocrine cancers. Journal of Clinical Oncology 29 2416-2423.

Janson ET, Sorbye H, Welin S, Federspiel B, Gronbaek H, Hellman P, Ladekarl M, Langer SW, Mortensen J, Schalin-Jantti C, et al. 2014 Nordic guidelines 2014 for diagnosis and treatment of gastroenteropancreatic neuroendocrine neoplasms. Acta Oncologica 53 1284-1297. (https://doi.org/10.3109/0284186X.2014.941999)

Kashyap R, Hofman MS, Michael M, Kong G, Akhurst T, Eu P, Zannino D \& Hicks RJ 2015 Favourable outcomes of (177) Lu-octreotate peptide receptor chemoradionuclide therapy in patients with FDG-avid neuroendocrine tumours. European Journal of Nuclear Medicine and Molecular Imaging 42 176-185. (https://doi. org/10.1007/s00259-014-2906-4)

Kayani I, Bomanji JB, Groves A, Conway G, Gacinovic S, Win T, Dickson J, Caplin M \& Ell PJ 2008 Functional imaging of neuroendocrine tumors with combined PET/CT using 68Ga-DOTATATE (DOTA-DPhe1,Tyr3-octreotate) and 18F-FDG. Cancer 112 2447-2455. (https://doi.org/10.1002/cncr.23469)

Kong G \& Hicks RJ 2019 Peptide receptor radiotherapy: current approaches and future directions. Current Treatment Options in Oncology 20 77. (https://doi.org/10.1007/s11864-019-0677-7)

Kong G, Thompson M, Collins M, Herschtal A, Hofman MS, Johnston V, Eu P, Michael M \& Hicks RJ 2014 Assessment of predictors of response and long-term survival of patients with neuroendocrine tumour treated with peptide receptor chemoradionuclide therapy (PRCRT). European Journal of Nuclear Medicine and Molecular Imaging 41 1831-1844. (https://doi.org/10.1007/s00259-014-2788-5)

Konukiewitz B, Schlitter A M, Jesinghaus M, Pfister D, Steiger K, Segler A, Agaimy A, Sipos B, Zamboni G, Weichert W, et al. 2017 Somatostatin receptor expression related to TP53 and RB1 alterations in pancreatic and extrapancreatic neuroendocrine neoplasms with a Ki67-index above 20\%. Modern Pathology 30 587-598.

Kwekkeboom DJ, Teunissen JJ, Bakker WH, Kooij PP, De Herder WW, Feelders RA, Van Eijck CH, Esser JP, Kam BL \& Krenning EP 2005 Radiolabeled somatostatin analog [177Lu-DOTA0,Tyr3]octreotate in patients with endocrine gastroenteropancreatic tumors. Journal of Clinical Oncology 23 2754-2762. (https://doi.org/10.1200/ JCO.2005.08.066)

Kwekkeboom DJ, De Herder WW, Kam BL, Van Eijck CH, Van Essen M, Kooij PP, Feelders RA, Van Aken MO \& Krenning EP 2008 Treatment with the radiolabeled somatostatin analog [Lu-177-DOTA(0), Tyr(3)] octreotate: toxicity, efficacy, and survival. Journal of Clinical Oncology 26 2124-2130.

Lamarca A, Walter T, Pavel M, Borbath I, Freis P, Nunez B, Childs A, Mcnamara MG, Hubner RA, Garcia-Carbonero R, et al. 2017 Design and validation of the GI-NEC score to prognosticate overall survival in patients with high-grade gastrointestinal neuroendocrine carcinomas. Journal of the National Cancer Institute 109 djw277. (https://doi.org/10.1093/jnci/djw277)

Leoncini E, Boffetta P, Shafir M, Aleksovska K, Boccia S \& Rindi G 2017 Increased incidence trend of low-grade and high-grade neuroendocrine neoplasms. Endocrine 58 368-379. (https://doi. org/10.1007/s12020-017-1273-x)

Liverani C, Bongiovanni A, Mercatali L, Foca F, Pieri F, De Vita A, Spadazzi C, Miserocchi G, Recine F, Riva N, et al. 2018 Grading of neuroendocrine carcinomas: correlation of (68)Ga. Disease Markers 2018 6878409. (https://doi.org/10.1155/2018/6878409)

Milione M, Maisonneuve P, Spada F, Pellegrinelli A, Spaggiari P, Albarello L, Pisa E, Barberis M, Vanoli A, Buzzoni R, et al. 2017 The clinicopathologic heterogeneity of Grade 3 gastroenteropancreatic neuroendocrine neoplasms: morphological differentiation and proliferation identify different prognostic categories. Neuroendocrinology 104 85-93. (https://doi.org/10.1159/000445165)

Mizuno Y, Kudo A, Akashi T, Akahoshi K, Ogura T, Ogawa K, Ono H, Mitsunori Y, Ban D, Tanaka S, et al. 2018 Sunitinib shrinks NET-G3 pancreatic neuroendocrine neoplasms. Journal of Cancer Research and Clinical Oncology 144 1155-1163. (https://doi.org/10.1007/s00432018-2636-2)

NCCN 2019 Neuroendocrine and adrenal tumors. NCCN Clinical Practice Guidelines in Oncology. Plymouth Meeting, PA, USA: NCCN. (available at: https://www.nccn.org/professionals/physician_gls/ default.aspx\#neuroendocrine)

Nicolini S, Severi S, Ianniello A, Sansovini M, Ambrosetti A, Bongiovanni A, Scarpi E, Di Mauro F, Rossi A, Matteucci F, et al. 2018 Investigation of receptor radionuclide therapy with (177) Lu-DOTATATE in patients with GEP-NEN and a high Ki-67 proliferation index. European Journal of Nuclear Medicine and Molecular Imaging 45 923-930. (https://doi.org/10.1007/s00259-017-3925-8)

Panzuto F, Rinzivillo M, Spada F, Antonuzzo L, Ibrahim T, Campana D, Fazio N \& Delle Fave G 2017 Everolimus in pancreatic neuroendocrine carcinomas G3. Pancreas 46 302-305. (https://doi. org/10.1097/MPA.0000000000000762)

Patel S, Othus M, Chae Y, Giles F, Hansel D, Singh P, Fontaine A, Shah M, Kasi A, Baghdadi T, et al. 20192019 A phase II basket trial of dual anti-CTLA-4 and anti-PD-1 blockade in rare tumors (Dart) S1609: the neuroendocrine cohort. Cancer Research. 79 (13 Suppl) abstact CT039. (https://doi.org/10.1158/1538-7445.AM2019-CT039)

Raj N, Valentino E, Capanu M, Tang LH, Basturk O, Untch BR, Allen PJ, Klimstra DS \& Reidy-Lagunes D 2017 Treatment response and outcomes of Grade 3 pancreatic neuroendocrine neoplasms based on morphology: well differentiated versus poorly differentiated. Pancreas 46 296-301. (https://doi.org/10.1097/MPA.0000000000000735)

Rogowski W, Wachula E, Gorzelak A, Lebiedzinska A, Sulzyc-Bielicka V, Izycka-Swieszewska E, Zolnierek J \& Kos-Kudla B 2019 Capecitabine and temozolomide combination for treatment of high-grade, welldifferentiated neuroendocrine tumour and poorly-differentiated https://erc.bioscientifica.com

https://doi.org/10.1530/ERC-19-0400 (c) 2020 Society for Endocrinology Published by Bioscientifica Ltd. Printed in Great Britain 
neuroendocrine carcinoma - retrospective analysis. Endokrynologia Polska 70 313-317. (https://doi.org/10.5603/EP.a2019.0010)

Rudisile S, Gosewisch A, Wenter V, Unterrainer M, Boning G, Gildehaus FJ, Fendler WP, Auernhammer CJ, Spitzweg C, Bartenstein P, et al. 2019 Salvage PRRT with 177Lu-DOTA-octreotate in extensively pretreated patients with metastatic neuroendocrine tumor (NET): dosimetry, toxicity, efficacy, and survival. BMC Cancer 19 788. (https://doi.org/10.1186/s12885-019-6000-y)

Sorbye H, Welin S, Langer SW, Vestermark LW, Holt N, Osterlund P, Dueland S, Hofsli E, Guren MG, Ohrling K, et al. 2013 Predictive and prognostic factors for treatment and survival in 305 patients with advanced gastrointestinal neuroendocrine carcinoma (WHO G3): the NORDIC NEC study. Annals of Oncology 24 152-160. (https://doi. org/10.1093/annonc/mds276)

Sorbye H, Strosberg J, Baudin E, Klimstra DS \& Yao JC 2014 Gastroenteropancreatic high-grade neuroendocrine carcinoma. Cancer 120 2814-2823. (https://doi.org/10.1002/cncr.28721)

Sorbye H, Baudin E \& Perren A 2018 The problem of high-grade gastroenteropancreatic neuroendocrine neoplasms: welldifferentiated neuroendocrine tumors, neuroendocrine carcinomas, and beyond. Endocrinology and Metabolism Clinics of North America $\mathbf{4 7}$ 683-698. (https://doi.org/10.1016/j.ecl.2018.05.001)

Sorbye H, Baudin E, Borbath I, Caplin M, Chen J, Cwikla JB, Frilling A, Grossman A, Kaltsas G, Scarpa A, et al. 2019 Unmet needs in highgrade gastroenteropancreatic neuroendocrine neoplasms (WHO G3). Neuroendocrinology 108 54-62. (https://doi.org/10.1159/000493318)

Strosberg JR, Coppola D, Klimstra DS, Phan AT, Kulke MH, Wiseman GA, Kvols LK \& North American Neuroendocrine Tumor Society (NANETS) 2010 The NANETS consensus guidelines for the diagnosis and management of poorly differentiated (high-grade) extrapulmonary neuroendocrine carcinomas. Pancreas 39 799-800. (https://doi.org/10.1097/MPA.0b013e3181ebb56f)

Strosberg J, El-Haddad G, Wolin E, Hendifar A, Yao J, Chasen B, Mittra E, Kunz PL, Kulke MH, Jacene H, et al. 2017 Phase 3 trial of (177)Lu-Dotatate for midgut neuroendocrine tumors. New England Journal of Medicine 376 125-135. (https://doi.org/10.1056/ NEJMoa1607427)

Sundin A, Arnold R, Baudin E, Cwikla JB, Eriksson B, Fanti S, Fazio N, Giammarile F, Hicks RJ, Kjaer A, et al. 2017 Enets consensus guidelines for the standards of care in neuroendocrine tumors: radiological, nuclear medicine and hybrid imaging. Neuroendocrinology $105212-$ 244. (https://doi.org/10.1159/000471879)

Tang LH, Basturk O, Sue JJ \& Klimstra DS 2016a A practical approach to the classification of WHO grade 3 (G3) well-differentiated neuroendocrine tumor (WD-NET) and poorly differentiated neuroendocrine carcinoma (PD-NEC) of the pancreas. American Journal of Surgical Pathology 40 1192-1202. (https://doi.org/10.1097/ PAS.0000000000000662)

Tang LH, Untch BR, Reidy DL, O'Reilly E, Dhall D, Jih L, Basturk O, Allen PJ \& Klimstra DS 2016 $b$ Well-differentiated neuroendocrine tumors with a morphologically apparent high-grade component: a pathway distinct from poorly differentiated neuroendocrine carcinomas. Clinical Cancer Research 22 1011-1017. (https://doi. org/10.1158/1078-0432.CCR-15-0548)

Thang SP, Lung MS, Kong G, Hofman MS, Callahan J, Michael M \& Hicks RJ 2017 Peptide receptor radionuclide therapy (PRRT) in European Neuroendocrine Tumour Society (ENETS) grade 3 (G3) neuroendocrine neoplasia (NEN) - a single-institution retrospective analysis. European Journal of Nuclear Medicine and Molecular Imaging 45 262-277. (https://doi.org/10.1007/s00259-017-3821-2)

Van Der Zwan WA, Brabander T, Kam BLR, Teunissen JJM, Feelders RA, Hofland J, Krenning EP \& De Herder WW 2019 Salvage peptide receptor radionuclide therapy with [(177)Lu-DOTA,Tyr(3)]octreotate in patients with bronchial and gastroenteropancreatic neuroendocrine tumours. European Journal of Nuclear Medicine and Molecular Imaging 46 704-717. (https://doi.org/10.1007/s00259-0184158-1)

Velayoudom-Cephise FL, Duvillard P, Foucan L, Hadoux J, Chougnet CN, Leboulleux S, Malka D, Guigay J, Goere D, Debaere T, et al. 2013 Are G3 Enets neuroendocrine neoplasms heterogeneous? Endocrine-Related Cancer 20 649-657. (https://doi.org/10.1530/ERC13-0027)

Walter T, Tougeron D, Baudin E, Le Malicot K, Lecomte T, Malka D, Hentic O, Manfredi S, Bonnet I, Guimbaud R, et al. 2017 Poorly differentiated gastro-entero-pancreatic neuroendocrine carcinomas: are they really heterogeneous? Insights from the FFCD-GTE national cohort. European Journal of Cancer 79 158-165. (https://doi. org/10.1016/j.ejca.2017.04.009)

Welin S, Sorbye H, Sebjornsen S, Knappskog S, Busch C \& Oberg K 2011 Clinical effect of temozolomide-based chemotherapy in poorly differentiated endocrine carcinoma after progression on first-line chemotherapy. Cancer 117 4617-4622. (https://doi.org/10.1002/ cncr.26124)

Yamaguchi T, Machida N, Morizane C, Kasuga A, Takahashi H, Sudo K, Nishina T, Tobimatsu K, Ishido K, Furuse J, et al. 2014 Multicenter retrospective analysis of systemic chemotherapy for advanced neuroendocrine carcinoma of the digestive system. Cancer Science 105 1176-1181. (https://doi.org/10.1111/cas.12473)

Yao J, Strosberg J, Fazio N, Pavel ME, Ruszniewski P, Bergsland E, et al. 2018 Activity and safety of spartalizumab (PDR001) in patients with advanced neuroendocrine tumors (NET) of pancreatic (Pan), gastrointestinal (GI), or thoracic (T) origin, and gastroenteropancreatic neuroendocrine carcinoma (GEP NEC) who have progressed on prior treatment. Annals of Oncology 29 (Supplement 8) abstract 13080.

Zhang J, Kulkarni HR, Singh A, Niepsch K, Muller D \& Baum RP 2019 Peptide receptor radionuclide therapy in Grade 3 neuroendocrine neoplasms: safety and survival analysis in 69 patients. Journal of Nuclear Medicine 60 377-385. (https://doi.org/10.2967/ jnumed.118.215848)

Received in final form 2 December 2019

Accepted 16 December 2019

Accepted Manuscript published online 17 December 2019
C) 2020 Society for Endocrinology Published by Bioscientifica Ltd. Printed in Great Britain 